II.

MIRRORS AND DOMES 


\title{
STEPS TOWARDS 8M HONEYCOMB MIRRORS V \\ A METHOD FOR POLISHING ASPHERES AS FAST AS F/1
}

\author{
J. R. P. Angel \\ Steward Observatory, University of Arizona
}

\section{ABSTRACT}

A method is proposed for polishing fast aspherics with a lap whose shape is continuously changed under computer control as it moves over a rigid mirror blank. The required changes of radius, astigmatism and coma in a circular lap are made with edge bending levers and tensioning members with screw actuators. This method of bending has been demonstrated in the laboratory.

\section{INTRODUCTION}

In a series of four preceding papers ${ }^{1-4}$ we have argued that an optimum diameter for large telescope mirrors is $8 \mathrm{~m}$, and have described the development of a method suitable for casting lightweight honeycomb glass blanks of this size. Rotation is used to preform the parabolic dish shape, and the $8 \mathrm{~m}$ size spinning furnace under development will allow the formation of curves as fast as

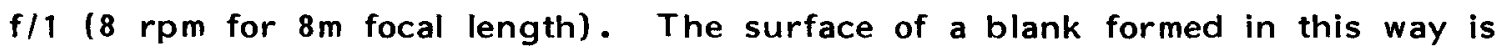
not of optical quality, but it is within a millimeter or so of the desired curve. Finishing to within a few microns RMS of a paraboloid can then be achieved rapidly with a numerically controlled precision diamond generator, such as the $8 \mathrm{~m}$ LOC at the Optical Sciences Center. 5

If $8 \mathrm{~m}$ telescopes were built with $\mathrm{f} / 1$ primaries, the moving structure and enclosing building (if any) would be considerably smaller, lighter and less expensive than for currently favored designs with $f / 2$ - $f / 3$ primaries. In a paper presented at this meeting. Epps, Angel, and Anderson ${ }^{6}$ give an optical design for an $f / 1$ paraboloidal primary. It has a Cassegrain focus at $f / 4$, which with the aid of a corrector of 3 fused silica elements realizes a flat field of 40 arcminutes, with excellent image quality and achromatic correction. The same paraboloidal primary, with a small chopping secondary, would give a good IR focus at reasonable focal ratio and plate scale.

The case for going to $f / 1$ primaries (Woolf, Angel, and Williams ${ }^{7}$ ) is strong. The question is, how do you figure such a fast large aspheric surface to the very high level of performance now needed for telescopes at the best sites? No practical method currently exists, and this is the reason that optical telescopes are as clumsy as they are.

Proceedings of the IAU Colloquium No. 79: "Very Large Telescopes, their Instrumentation and Programs", Garching, April 9-12, 1984. 
The conventional process of making accurate optical surfaces, still basically the same as used by Galileo and Newton, makes use of the fact that two rigid bodies rubbed against each other with abrasive tend to form a spherical boundary. This powerful principle allows spherical lenses and mirrors to be polished easily to very high precision, small fractions of the wavelength of light, with simple equipment. There would be little difficulty in polishing a fast $\mathrm{f} / 1$ sphere of $8 \mathrm{~m}$ diameter.

When lapping and polishing is carried out with a lap that is somewhat flexible or small compared to the work, then the tendency to make a sphere is weakened. In these circumstances, the optician has some latitude to figure surfaces that are modestly aspheric, using an iterative process. The surface is worked, its shape is measured and its error from the desired figure determined, and then the surface is worked again to reduce the error. In this way paraboloidal mirrors of $2.5 \mathrm{~m}$ diameter as fast as $f / 2.2$ have been made. Examples are the diffraction limited mirrors for the Space Telescope program made by Perkin Elmer and Kodak, and the series of mirrors made by Norman Cole for ground-based telescopes. The same methods can be pushed to make somewhat faster mirrors, but difficulty increases rapidly with decreasing focal ratio. This is because the variations in curvature across a parabolic mirror of given diameter increases as the inverse cube of the focal ratio. If a lap, either by being small or flexible, can accommodate the desired variations in curvature of the asphere, it can also accommodate undesired ones. Thus as focal ratio is reduced, zones and ripples appearing on smaller and smaller spatial scales rapidly become more difficult to control. One must rely more and more heavily on the interactive figuring cycle, explicitly tailoring the polishing to match in detail the measured errors.

The problem of correcting errors is made all the more difficult because a flexible lap will in fact never be a very good fit to an aspheric surface. The only forces acting on the lap available to change is curvature are its weight and the polishing pressure reaction from the glass. These forces cannot apply any moment at the edge of the lap, so its curvature here cannot be changed, and will not fit correctly.

These considerations led us to the idea of using a stiff lap specially fitted with internal force actuators, so that its curvature can be explicitly changed to accommodate the desired asphere. Imagine the bow of a bow and arrow, fitted with a motor driven linear actuator instead of an arrow. By driving the motor we can change the curvature of the bow, without the application of any external force. Suppose now that a stiff polishing lap could be similarly fitted 
with strong actuators, so its shape could be accurately changed at will from that of an on-axis parabola to an off-axis segment of the same parent. If the shape were changed continuously during polishing strokes and rotation, so that at any position it conformed to the desired parabola, then the polishing of the parabola would become similar to that of polishing a sphere. A preliminary discussion of this method is given by Angel and Parks. ${ }^{8}$

There are two important advantages in this active bending approach, compared to that of using a passive flexible lap. Firstly, the active lap can be much stiffer, and give much better supression of ripples. This is because strong forces can be applied by internal actuators. The limit is set by the tensile strength of the lap material, which for steel is around $10^{9} \mathrm{~N} / \mathrm{m}^{2}$. Internal stresses in a passive lap are limited by the polishing pressure, and depending on lap geometry are unlikely to exceed $10^{6} \mathrm{~N} / \mathrm{m}^{2}$. Secondly, the active lap can make use of actuators to apply bending moments at the edge of the lap. These allow for the curvature to be changed across the whole face of the lap, with no inherent problem at the edges. The potential exists for very accurate matching of the desired shape changes.

There is also an important difference in the role played by the computer in computer controlled polishing with small laps (CCP) and in active stressed lap polishing. In CCP, the lap motion is programmed differently for each iteration, in response to a detailed figure error map. The active lap actuators, by contrast, are programmed to bend always in accordance with the final desired figure. The continuous frenetic activity of the computer in controlling the lap proceeds essentially independently from that of figuring the large scale errors.

\section{THE MECHANICAL CONFIGURATION FOR AN ACTIVE LAP}

We now consider what changes of shape of the lap are required, and how best to achieve them. It is convenient to express the shape of an aspheric near any point as the distance $z$ between its surface and a spherical reference surface which touches at that point. Let the aspheric have conic constant $k$, the reference sphere have radius $R$ equal to the vertex radius of the asphere, and touch at a distance $h$ from the axis of the asphere. Then $z$ is given by

$$
z \simeq k h^{2} r^{2}(2+\cos 2 \theta) / 4 R^{3}+k h r^{3} \cos \theta / 2 R^{3}+k r^{4} / 8 R^{3}
$$

where $r$ is measured from the point of contact, $\theta$ from the direction of the radius vector from the axis of the asphere. Following Parks, ${ }^{9}$ we express $z$ as a superposition of elementary surface. The change in shape $\Delta z$ as the point of 
contact is moved over the asphere requires only the three terms of focus change, astigmatisn and coma. If a lap of radius $a$ is used, and we set $r / a=\rho$, then

$$
\begin{aligned}
\Delta z & \simeq k a^{2} h^{2} / 4 R^{3}\left(2 \rho^{2}-1\right) & & \text { (focus) } \\
& +k a^{2} h^{2} / 4 R^{3}\left(\rho^{2} \cos 2 \theta\right) & & \text { (astigmatism) } \\
& +k a^{3} h / 6 R^{3}\left(3 \rho^{3}-2 \rho\right) \cos \theta & & \text { (coma) }
\end{aligned}
$$

Terms in tilt and piston error are omitted.

These three terms can be induced in a uniform circular plate simply by the application of the correct distribution of bending moment around the edge of the plate, and appropriate stabilizing edge shear forces. No forces distributed across the face of the lap are needed, so the polishing pressure can be uniform and chosen freely on polishing considerations alone. We have devised an implementation for lap bending that involves only levers and tensioning members, with no need for any reaction forces except within the lap itself. This is shown schematically in Figure 1. Levers whose length are about $1 / 3$ of the lap

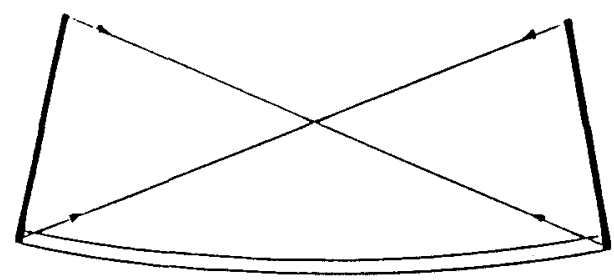

(a) change of radius

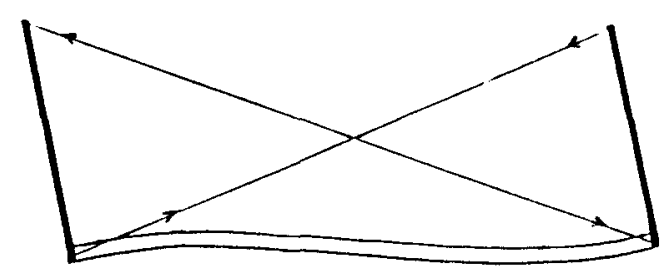

(b) coma

Figure 1. Schematic of bending method.

diameter are arranged around the circumference, sticking up perpendicular to the lap. Each is connected by a cable to a point on the lap surface diametrically opposite, and a motor actuator is used to vary each cable tension. When all the rods are tensioned uniformly, the lap radius is changed (Figure 1a). Astigmatism is induced by increasing the tension in opposite groups of levers. A coma term can be added by increasing the tension on neighboring levers and decreasing it on the opposite group. This is shown schematically in Figure 1b, where for clarity we show a linking member in compression. In practice, an offset of enough uniform tension is applied, so that changes in shape never reauire anything but changes in tension on the different levers.

\section{STATIC DEFLECTIONS OF A TEST LAP MEASURED BY HOLOGRAPHY}

To check that the proposed stressing scheme will work in practice, we have built a model from a circular disc of glass. For convenience of testing by holography, this was given a concave mirror surface. Details of the model are shown in Figure 2. The glass disc is $33.3 \mathrm{~cm}$ in diameter and $2.5 \mathrm{~cm}$ thick, plano concave with a radius of $3 \mathrm{~m}$. 24 invar blocks are epoxied to the circumference, 


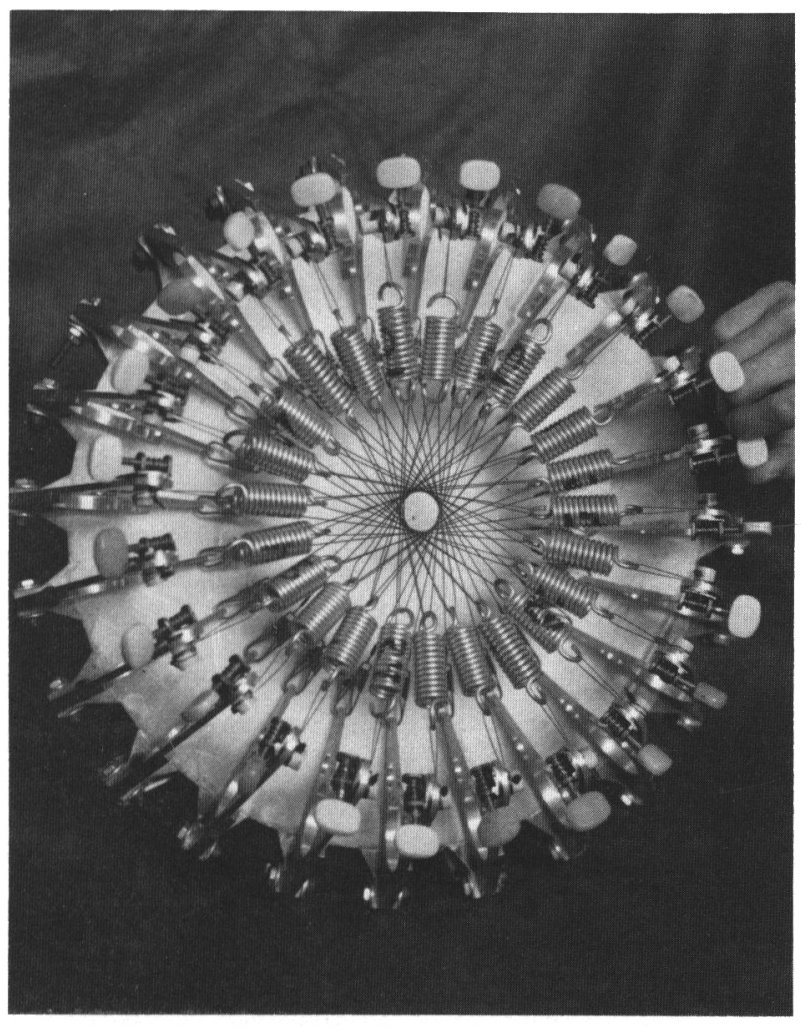

(a) each carrying an aluminum lever as shown. The lever has two arms, the upper one fitted with a guitar key to tighten the tensioning cable. The lower arm anchors cables close to the back face of the disc, and is set so the line of action is through the middle of the disc edge. (In a metal lap the lower arm would be eliminated. It was used simply to avoid working the glass disc.) The cables, of $65 \mathrm{lb}$ test Toothy Critter fishing line, act through springs. Spring constants were determined before assembly, so known forces could be applied by setting spring lengths with callipers. Each spring is connected not to the lower arm diagonally opposite, but with a $V$ cable to the lower arms adjacent.

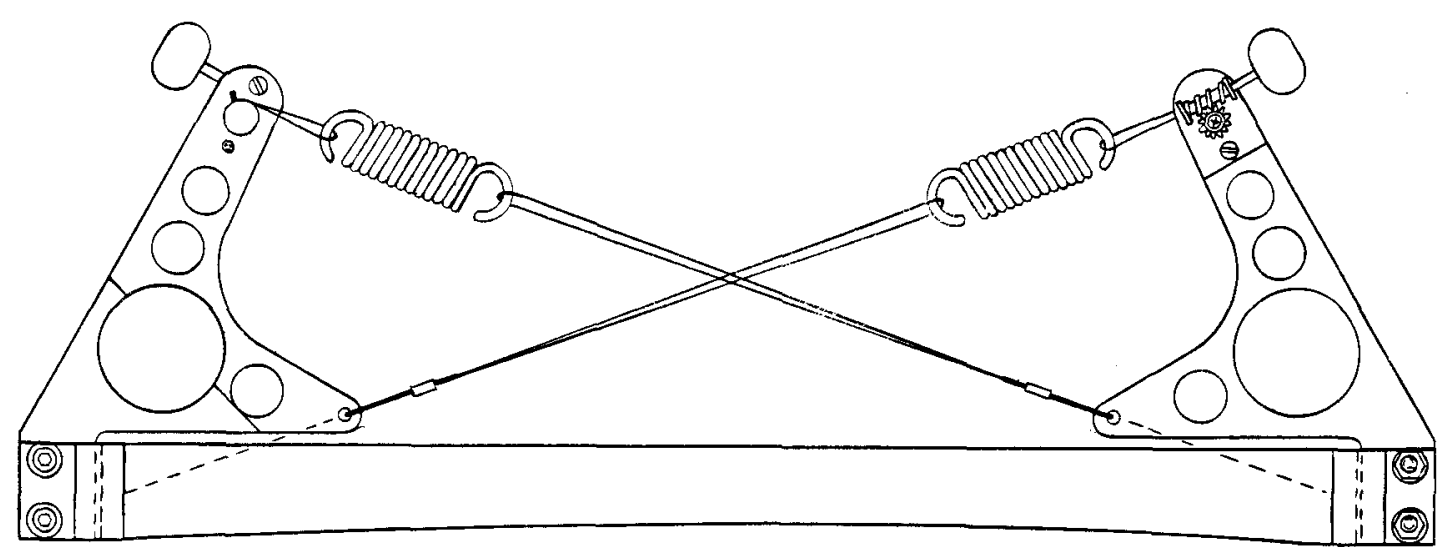

(b)

Figure 2. (a) Photograph of the test model; (b) cross section. 
Bending of the surface of the model was measured by holography by Phil Lam at the Optical Sciences Center. A hologram is used to record the wavefront coming from the surface in one state of stress, then after changing the stress the new and reconstructed wavefronts are allowed to interfere, showing directly the change in shape. Fringes formed by the HeNe laser show contour intervals of the deflection in units of $0.32 \mu$.

An important characterization of the bending geometry is the influence function or bending caused by tensioning one cable alone. This is given in the interferogram of Figure 3, which shows the bending for a force of $9.2 \mathrm{~kg}$. The

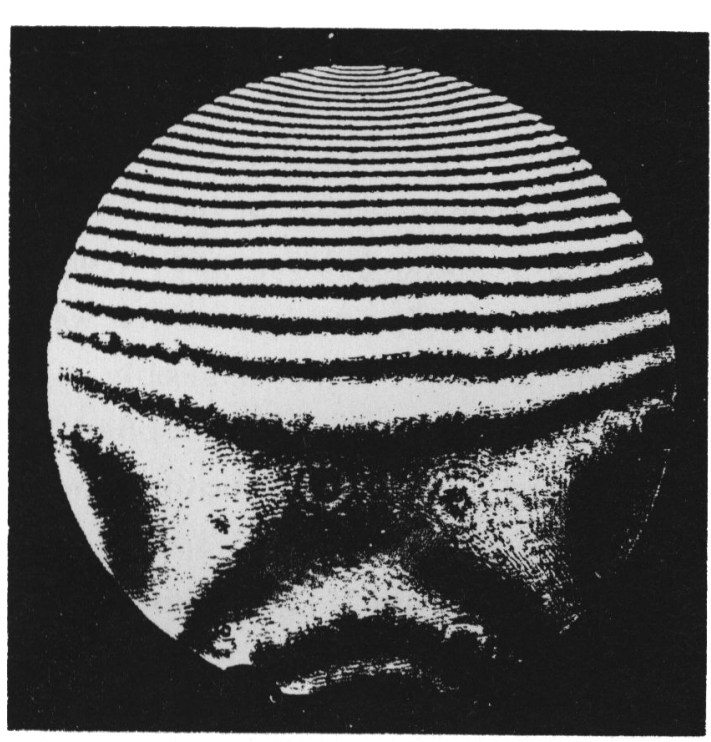
bending caused by any combination of cable tensions is given simply by a linear superposition of the 24 individual contributions. Similarly, if a given bending displacement is desired, then the combination of cable tensions that best reproduces it can be determined analytically by the method of least squares, once the influence function is known.

Let the influence function for a single cable acting at $\theta=0$ be $l_{0}(r, \theta)$. The remaining 23 functions are given by $I_{n}=I_{0}(r, \theta-n \pi / 12)$. Now let the best fit to the desired bending function, $F$, be given by

$$
F(r, \theta)=\sum_{n=0}^{23} a_{n} I_{n}+a_{24} \cos \theta+a_{25} \sin \theta+a_{26}
$$

Thus $a_{0}$ to $a_{23}$ give the cable tensions, $a_{24}$ and $a_{25}$ the tilt and $a_{26}$ the piston terms. The RMS error of this best fit is then given by

$$
E^{2}=\int\left(F-\sum_{N=0}^{23} a_{n}{ }^{\prime} n-a_{24} \cos \theta-a_{25} \sin \theta-a_{26}\right)^{2} r d r d \theta / A \text {. }
$$

where $A$ is the area of the lap. The best fit value of the a coefficients is found by solving twenty-six simultaneous equations, obtained by setting equal to zero the partial derivatives of $E^{2}$ with respect to each coefficient. 
In this way coefficients for focus change, coma and astigmatism were computed, using as input the influence function of Figure 3. The integral over the disc was done by summing over seven rings equally spaced in radius, at points every $7-1 / 2^{\circ}$ on each ring. In Figure 4 are given the calculated forces for the functions of focus change, astigmatism and coma, normalized to unit force producing the first function, $2 p^{2}-1$. The fourth function is appropriate for bending a $1 / 3$ diameter lap to fit at the edge of an $f / 1$ parabola. The RMS error of the computed best fits is given also in Figure 4. It is small, and a map of the errors shows edge fluting to be a major component of the error.
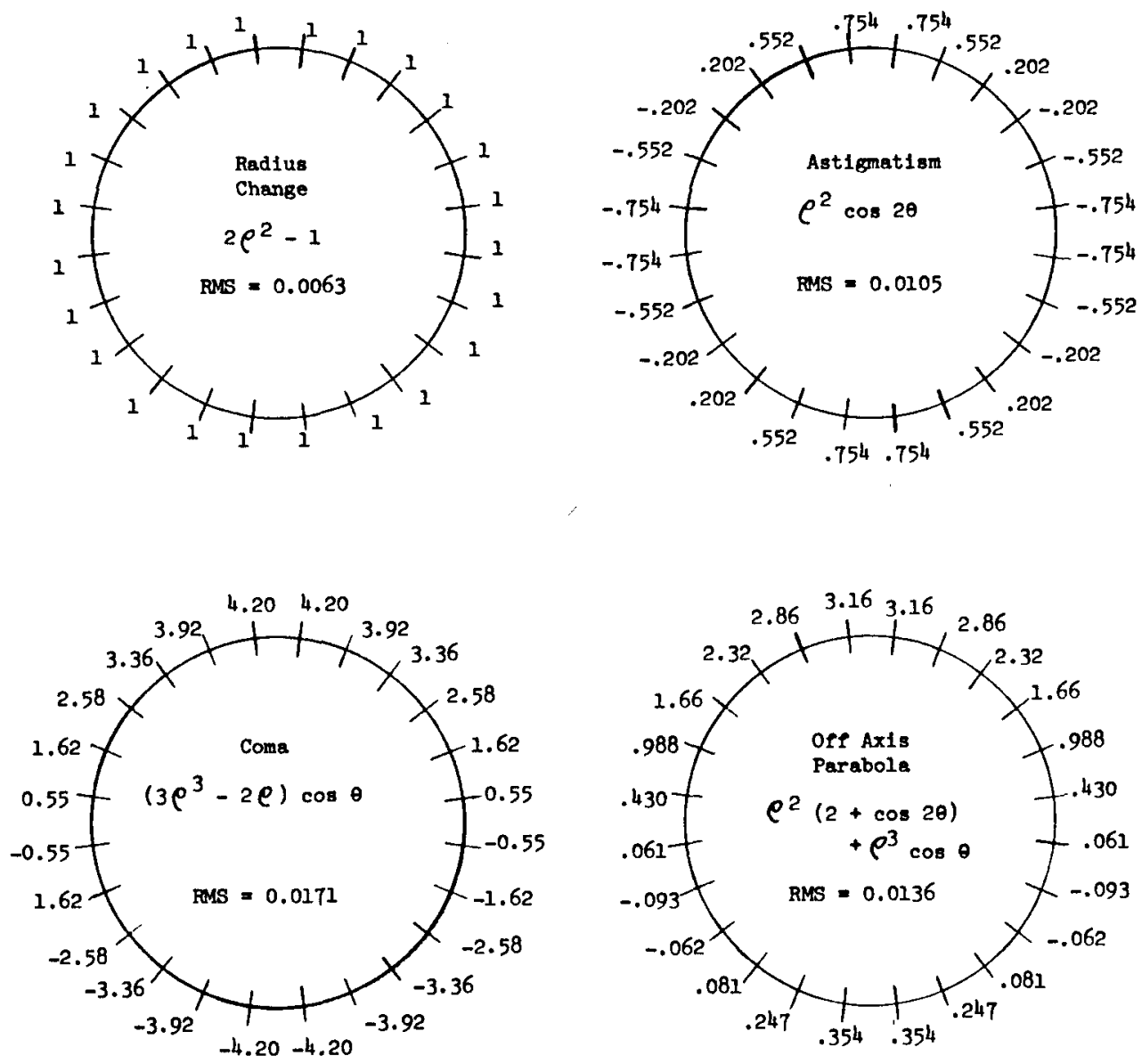

Figure 4. Pattern of actuator forces needed to induce the functions given. Solutions computed from the measured influence function.

To check the practical realization of bending to a specific function, we obtained the interferograms in Figure 5. Figure $5 \mathrm{a}$ was obtained by exposing the hologram initially with no stress, then tightening each cable to the same $1.46 \mathrm{~kg}$ force. The plate shows change of radius, with no significant edge fluting in practice. Figure $5 \mathrm{~b}$ was obtained by exposing the hologram with the forces of $4 \mathrm{c}$ 
needed to produce coma plus a constant offset. The springs were then adjusted to give simply the constant offset force, and the interferogram obtained. As can be seen, the change of shape is coma, as desired, and again there is very little edge fluting. We believe that errors in the influence function measurement are responsible for the poorer computed fits. It seems also that in practice the lack of strictly uniform thickness in the disc has not much affected the bending. Our conclusion is that the desired shape changes can be accurately induced with the 24 actuator system.

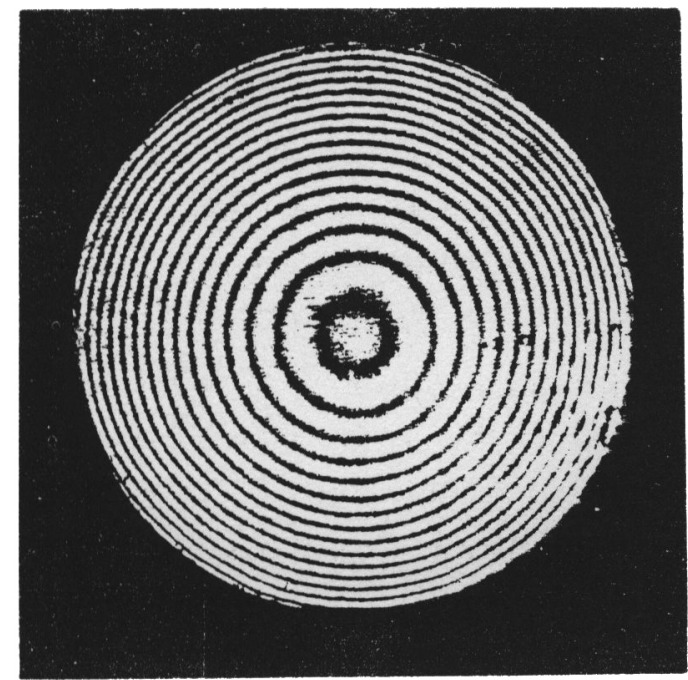

(a)

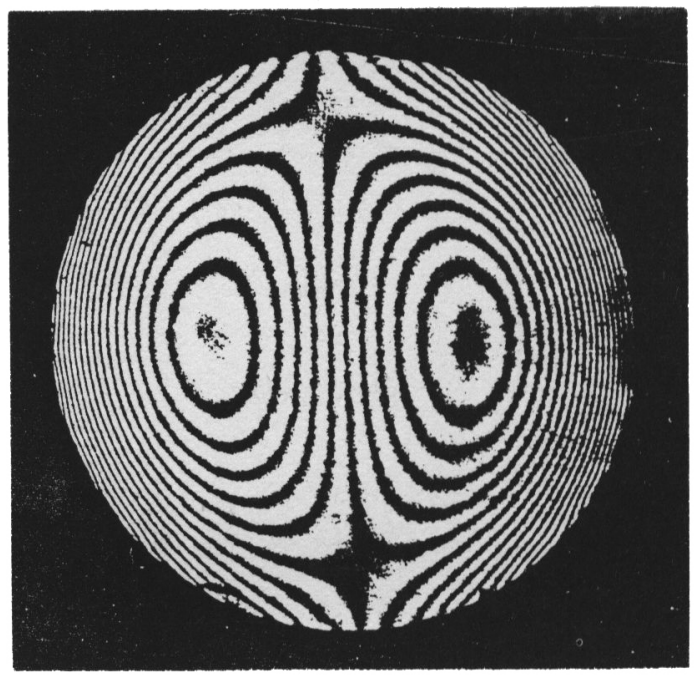

(b)

Figure 5. Bending induced in the test lap. (a) focus changes; (b) coma.

\section{A COMPARISON OF STRESSED LAP AND STRESSED MIRROR POLISHING}

Stessed lap polishing is complementary to those methods in which the glass mirror itself is stressed and polished with a rigid lap. The goal is the same, to modify the natural tendency of polishing to form spheres, without losing the advantage of smoothness. While polishing glass in a stressed state has long been used for the manufacture of Schmidt plates, it has recently been used to demonstrate the manufacture of off axis parabolic segments. This followed the important understanding by Lubliner and Nelson ${ }^{10}$ that such a segment could be deformed into a sphere. Indeed, it is because of this work, and its realization in practice by Nelson et al. ${ }^{11}$ and by $B$ arr et al. ${ }^{12}$ that we were sure that the similar deformation of a lap would be possible, with high accuracy.

An advantage of stressing the glass rather than the lap is that only static deflections are needed and glass is more perfectly elastic than metals.' However, the correct deflection for a paraboloid is more difficult to achieve in the glass than in the lap. The lap is required to change its shape from one 
part of a paraboloid to another, while the glass must be bent from a paraboloid to a sphere. The latter involves curvature varying quadratically with radius, in addition to the uniform and linear changes needed for the lap. The quadratic term (spherical aberration) cannot be achieved with edge moments, and requires a combination of edge shear and uniform pressure across the blank. Thus correct stressing of the glass cannot use the simple system mentioned earlier, but requires levers that apply specific shear and moment forces at the edge, together with a uniform support system.

A further critical difference is that stressed mirror polishing places strong constraints on the primary mirror substrate. By contrast, stressed lap polishing allows the use of stiff lightweighted blanks of any size and thickness, even for the fastest mirrors. Glass substrates to be stressed cannot be very large, so a large primary must be built up from separately figured circular segments. If figured circular segments are to be cut and fitted together as hexagons or keystones, then a the substrate will also be required to be unusually free of internal strain. The faster the focal ratio the more segments there must be, and the thinner and more flexible they must get, to avoid breaking the glass. Internal bending stresses vary as thickness $/ F^{3}$. Thus to bend a glass segment like that described in these proceedings by $B$ arr to $f / 1$ instead of $\mathrm{f} / 1.75$, and to keep within the same internal stress limit, it would have to be only $1.5 \mathrm{~cm}$ thick. Based on a safe upper limit of $7 \times 10^{6} \mathrm{~N} / \mathrm{m}^{2}$, a glass segment of an $8 \mathrm{~m} \mathrm{f} / 1$ paraboloid would have to be no more than $3.5 \mathrm{~cm}$ thick. These values are uncomfortably thin, and the focal ratio of $f / 1.75$ adopted for the California $10 \mathrm{~m}$ telescope is probably close to the practical limit for the method. No corresponding restriction on focal ratio applies to the stress lap method, since the flexibility needed in the lap is not carried over in to the mirror.

\section{FIGURING AN $8 \mathrm{~m}$ F/1 PARABOLOID}

We assume that the starting point would be a substrate generated to within a few microns RMS of the paraboloid. The first step is to lap off the surface damage layer while reducing the surface error. An active stressed lap of $2 \mathrm{~m}$ diameter is proposed, which would need bending of about $2 \mathrm{~mm}$ peak to valley as it was translated and rotated over the $8 \mathrm{~m}$ surface. The characteristic period for cycling stress in the lap would be a few seconds, and we envisage the controlling computer would update the actuator force pattern about every millisecond, to keep errors at or below the $0.1 \%$ level. The strokes would be chosen to minimize surface error as monitored with a $10 \mu$ interferometer. When fine grinding was complete, the same active lap would be faced with pitch and 
the surface polished out, now with a HeNe interferometer measuring the surface. Figuring would proceed as far as possible with the $2 \mathrm{~m}$ lap. If this could not achieve the desired quality, then $1 \mathrm{~m}$ or even $1 / 2 \mathrm{~m}$ active laps could be used to finish up. These would require only $0.5 \mathrm{~mm}$ and $0.13 \mathrm{~mm}$ peak to valley bending respectively.

We envisage that a rather stiff lap would be used, with maximum forces per cable of order $1000 \mathrm{~kg}$. Steel is probably the preferred material, because of its superior elastic properties. In order to give the lap high stiffness without excessive weight a honeycomb or ribbed structure may be required. Analysis and experiment will be needed to design such a lap that bends as a disc of uniform thickness under the edge moments.

\section{CONCLUSION}

Stressed lap polishing would seem to be an idea whose time has come. It is a method that would not be possible without a large number of accurate servo systems and a dedicated powerful computer on line, but this is now quite practical. It does not lend itself easily to small scale optics, but there is now a need for very large mirrors. It comes into its own for surfaces that depart strongly from spheres, and there is now this need also.

I am grateful to Phil Lam for setting up and running the holographic interferometer, to Bob Parks for introducing me to the art of mirror polishing, and to Dan Watson and Sterling Kopke for their assistance in making the "mirror harp" and to Sue Mitchell for assistance in running computer fits. This work is supported by NASA under grant \# NAGW 121.

\section{REFERENCES}

1. Angel, J. R. P., and Woolf, N. J. 1983, Annals of N.Y. Acad. Sci., 422, 163.

2. Hill, J. M., and Angel, J. R. P. 1983, Proc. SPIE, 380, 100.

3. Angel, J. R. P., and Hill, J. M. 1983, Proc. SPIE, 444, 184.

4. Angel, J. R. P., Woolf, N. J., Hill, J. M., and Goble, L. 1983, Proc. SPIE, 444, 194 .

5. Shannon, R. R., and Parks, R. E., Proc. SPIE, 444, 271.

6. Epps, H. W., Angel, J. R. P., and Anderson, E. R. 1984, these proceedings.

7. Woolf, N. J., Angel, J. R. P., and Williams, J. T. 1984, these proceedings.

8. Angel, J. R. P., and Parks, R. E., 1984, OSA Optical Fabrication and Testing Workshop Technical Notebook, Monterey, California.

9. Parks, R. E. 1980, OSA Optical Fabrication and Testing Workshop Technical Notebook, p. 139, Falmouth, Massachusetts. 
10. Lubliner, J., and Nelson, J. E. 1980, Appl. Opt. 19, 2332.

11. Nelson, J. E., Gabor, G., Hunt, L. K., Lubliner, J., and Mast, T. S. 1980, Applied Optics, 19, 2341.

12. Barr, L. D., Gillette, P. E., and Shu, K.-L. 1983, Proc. SPIE, 444, 262. 